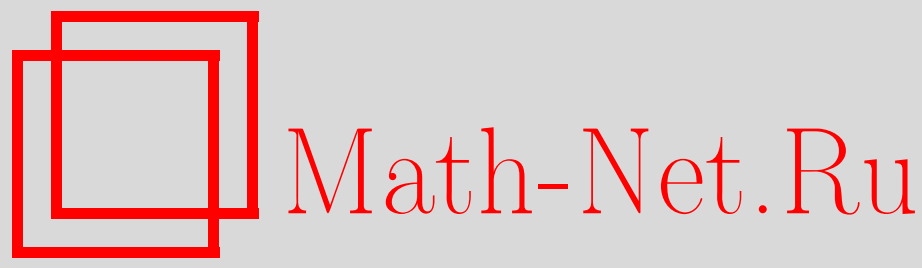

А. В. Самохин, Об уравнении Бюргерса с периодическими граничными условиями на интервале, ТМФ, 2016, том 188, номер 3, 470-476

DOI: https://doi.org/10.4213/tmf9075

Использование Общероссийского математического портала Math-Net.Ru подразумевает, что вы прочитали и согласны с пользовательским соглашением http://www.mathnet.ru/rus/agreement

Параметры загрузки:

IP : 52.23 .180 .231

26 апреля 2023 г., 13:51:33

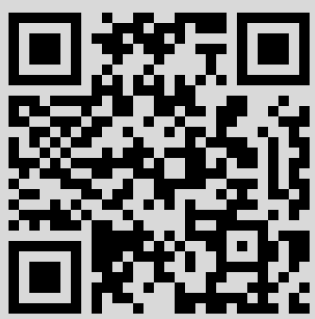




\section{ОБ УРАВНЕНИИ БЮРГЕРСА С ПЕРИОДИЧЕСКИМИ ГРАНИЧНЫМИ УСЛОВИЯМИ НА ИНТЕРВАЛЕ}

Исследуются асимптотики уравнения Бюргерса на конечном интервале с периодическим возмущением на границе. Уравнение описывает диссипативную среду, поэтому начальный постоянный профиль переходит в волну с уменьшающейся амплитудой. В случае малой вязкости асимптотический профиль выглядит, как пила (с периодическими разрывами производной), наподобие известного решения Фея для полупрямой, однако имеет некоторые новые свойства.

Ключевые слова: пилообразные решения, инвариантные решения, начально-граничная задача, асимптотики.

DOI: $10.4213 / \operatorname{tmf} 9075$

\section{1. ВВЕДЕНИЕ}

Решения уравнения Бюргерса в течение десятилетий были предметом интенсивного изучения, однако задачи ставились на прямой или полупрямой (для пространственой переменной) [1]-[3]. В последнее время исследования сосредоточились на таких аспектах, как критические точки, градиентные катастрофы, разрывы и т. п. [4]-[6].

В настоящей работе исследуются свойства решений уравнения Бюргерса

$$
u_{t}=\varepsilon^{2} u_{x x}-2 u u_{x}
$$

на конечном интервале $x \in[0, L]$. В этом случае начально-граничные условия выглядят следующим образом:

$$
u(x, 0)=f(x), \quad x \in[a, b], \quad u(a, t)=l(t), \quad u(b, t)=r(t) .
$$

В частности, начально-граничная модель для периодического возмущения на левом конце интервала имеет вид

$$
u(x, 0)=u_{0}, \quad u(0, t)=a+b \sin (\omega t), \quad u_{x}(L, t)=0 .
$$

* Московский государственный технический университет гражданской авиации, Москва, Россия. E-mail: samohinalexey@gmail.com 


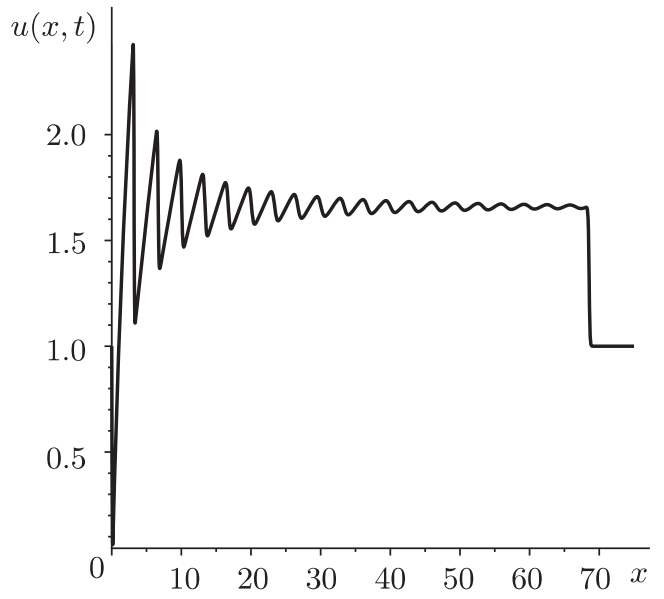

a

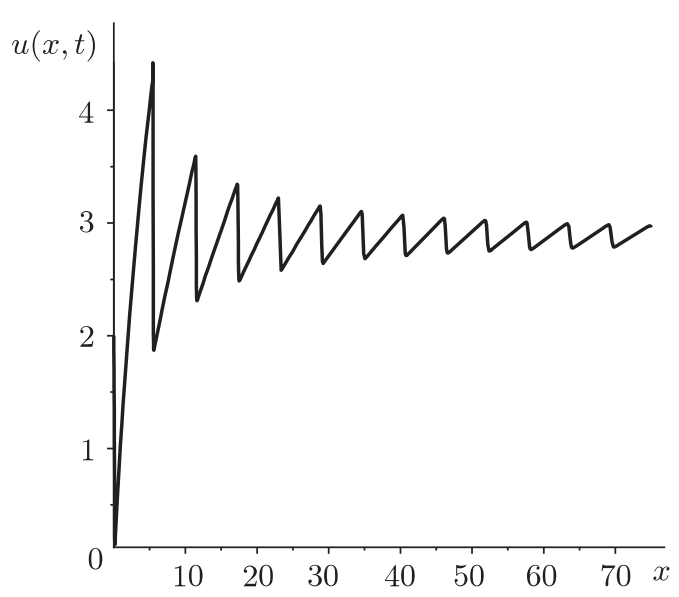

6

Рис. 1. Решение уравнения Бюргерса на полупрямой при $\varepsilon=0.2, t=25$ (a) и при $\varepsilon=0.15, t=25$ (б).

При $L=\infty$ асимптотика решения этой задачи совпадает с хорошо известным решением Фея [1]. В частности, $\lim _{x \rightarrow \infty} u(x, t)=a$, что равно среднему значению решения на полупрямой $x>0$.

Мы показываем, что это не так при $L<\infty$. Решение сохраняет пилообразный вид, но его среднее по интервалу $[0, L]$ отличается от $a$ и зависит также от амплитуды возмущения $b$. Кроме того, обсуждается взаимодействие между возмущениями с различающимися частотами.

\section{2. ПИЛООБРАЗНЫЕ ВОЛНЫ}

Для задачи на полупрямой $x \geqslant 0$ и периодического возмущения в точке $x=0$ вида $u(0, t)=u_{0}+b \sin (\omega t)$ асимптотика соответствующего решения такова [1]:

$$
u=\frac{a}{R} \sum_{n=1}^{\infty} \frac{\sin (n \theta)}{R \operatorname{sh}(n(1+X) / 2)} ;
$$

здесь $R$ - число Рейнольдса, $\theta=\omega\left(t-x / u_{0}\right)$. График такого решения обычно имеет форму, подобную представленной на рис. 1а. Можно заметить два эффекта вязкости. Во-первых, амплитуда колебаний экспоненциально убывает и $\lim _{x \rightarrow \infty} u=u_{0}$. Во-вторых, разрывы производной $u_{x}$ сконцентрированы в окрестности источника возмущения (вблизи $x=0$ ) и быстро исчезают при больших $x$.

Такие нелинейные эффекты широко применяются на практике (например, в медицине, где пилообразные волны используются для разрушения почечных камней).

2.1. Развитие пилообразных решений. Рассмотрим задачу

$$
\begin{gathered}
u_{t}=\varepsilon^{2} u_{x x}-2 u u_{x}, \\
u(x, 0)=1, \quad u(0, t)=1+2 \sin (2 \pi t), \quad u_{x}(L, t)=0,
\end{gathered}
$$



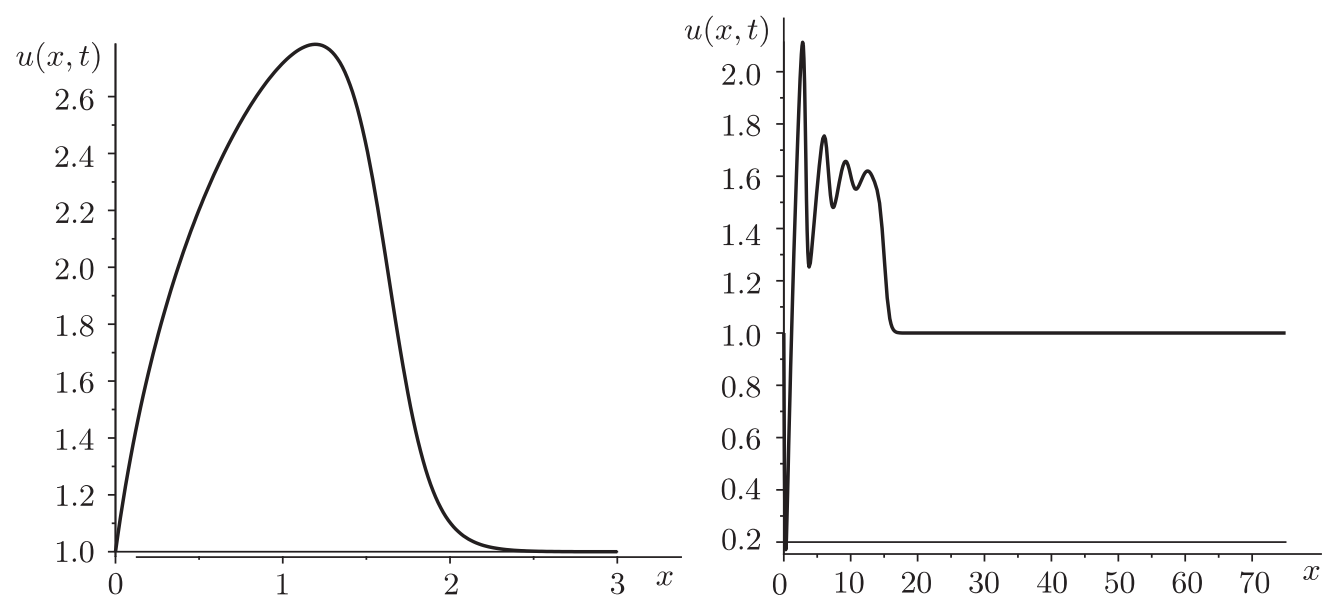

a

б
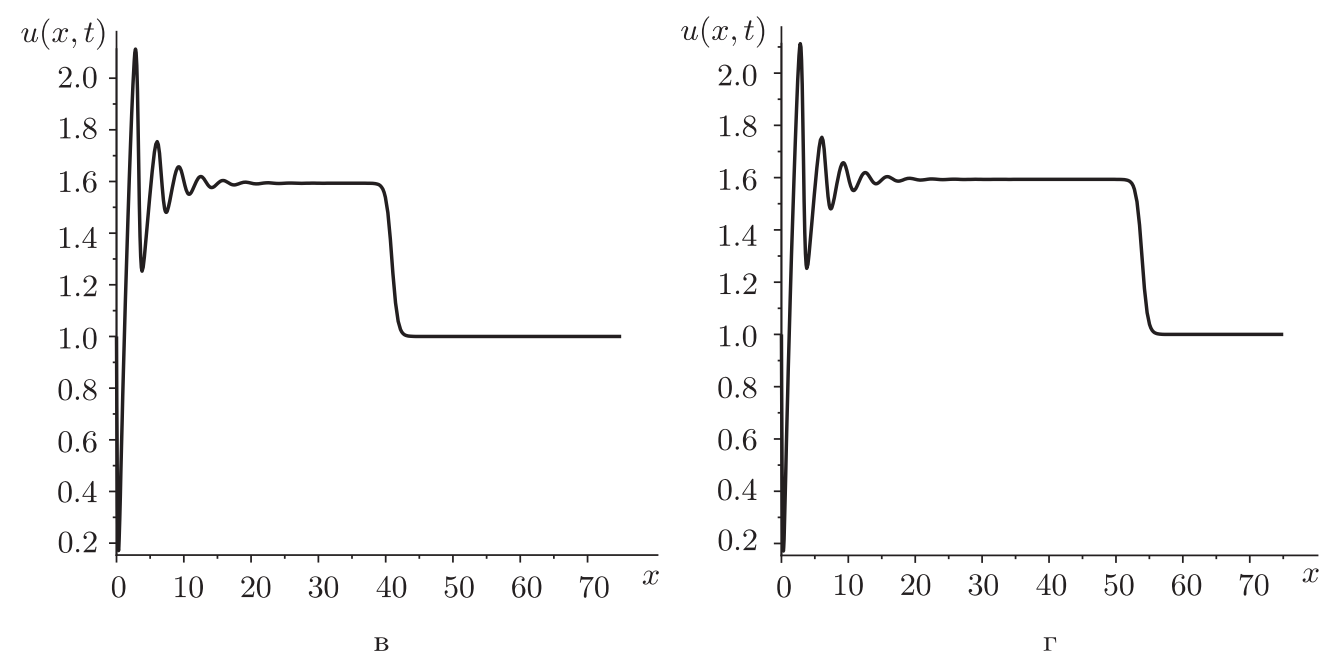

Рис. 2. Решение уравнения Бюргерса на интервале $[0, L]$ при $t=0.5$ (a), при $t=5$ (б), при $t=15$ (в) и при $t=20$ (г).

где $\varepsilon=0.5$ и $L=75$. Рис. 2 демонстрируют шаги перехода от постоянного начального условия к пилообразному профилю. Вязкость, соответствующая $\varepsilon=0.5$, довольно велика, и зубья пилы видны лишь в непосредственной близости от источника возмущения на левом конце интервала; по мере того как нарастает пространственное угасание, формируется уединенная ударная волна, движущаяся к правому концу.

Рис. 1 иллюстрирует случай $\varepsilon=0.2$ для начально-граничной задачи (2). Для меньшей вязкости пилообразная форма решения сохраняется на большем интервале значений $x$, что видно на рис. 16, который отвечает $\varepsilon=0.15$ и следующим начальнограничным условиям:

$$
u(x, 0)=2, \quad u(0, t)=2+3 \sin (2 \pi t), \quad u_{x}(75, t)=0 .
$$


График на рис. 1а дает зрительное представление о процессе постепенной трансформации острых зубьев с почти вертикальными передними краями в гладкие покатые осцилляции.

\section{3. ОЦЕНКИ ДЛЯ АСИМПТОТИК АМПЛИТУД И СКОРОСТИ}

Поскольку уравнение Бюргерса может быть переписано в форме закона сохранения $u_{t}=\left(\varepsilon^{2} u_{x}-u^{2}\right)_{x}$, мы имеем

$$
\oint_{\partial \mathcal{D}}\left[u d x+\left(\varepsilon^{2} u_{x}-u^{2}\right) d t\right]=0
$$

где $\mathcal{D}$ - прямоугольник $\{0 \leqslant x \leqslant L, 0 \leqslant t \leqslant T\}$, при этом интеграл можно переписать таким образом:

$$
\begin{aligned}
\int_{0}^{L} u(x, 0) d x & +\int_{0}^{T}\left[\varepsilon^{2} u_{x}(L, t)-u^{2}(L, t)\right] d t+ \\
& +\int_{L}^{0} u(x, T) d x+\int_{T}^{0}\left(\varepsilon^{2} u_{x}(0, t)-u^{2}(0, t)\right) d t=0 .
\end{aligned}
$$

Пусть $A$ обозначает среднее значение решения на отрезке $[0, L]$; из-за гасящего эффекта вязкости при достаточно больших $L$ и $T$ решение становится постоянным, $u(L, T)=A$. Используя условие $u_{x}(L, t)=0$, выводим

$$
u_{0} L-A^{2} T-A L-\int_{0}^{T}\left(\varepsilon^{2} u_{x}(0, t)-u^{2}(0, t)\right) d t=0 .
$$

Поделив на $T$, получаем квадратное уравнение для $A$ :

$$
-A^{2}-A \frac{L}{T}-\frac{1}{T} \int_{0}^{T}\left(\varepsilon^{2} u_{x}(0, t)-u^{2}(0, t)\right) d t+u_{0} \frac{L}{T}=0 .
$$

Поскольку интегралы от синуса и косинуса по периоду равны нулю, для $T \gg 0$ получим

$$
\frac{1}{T} \int_{0}^{T}\left(\varepsilon^{2} u_{x}(0, t)-u^{2}(0, t)\right) d t+u_{0} \frac{L}{T} \approx-u_{0}^{2}-\frac{a^{2}}{2},
$$

откуда

$$
-A^{2}-A k+u_{0}^{2}+\frac{a^{2}}{2}+u_{0} k=0
$$

где $k=L / T$. Таким образом, $A=\left(-k+\sqrt{k^{2}+4 u_{0}^{2}+2 a^{2}+4 u_{0} k}\right) / 2$. В результате имеем

$$
\lim _{T \rightarrow \infty} A=\lim _{k \rightarrow 0} A=\sqrt{u_{0}^{2}+\frac{a^{2}}{2}}, \quad \lim _{L \rightarrow \infty} A=\lim _{k \rightarrow \infty} A=u_{0},
$$

как и должно быть для задачи на полупрямой. 


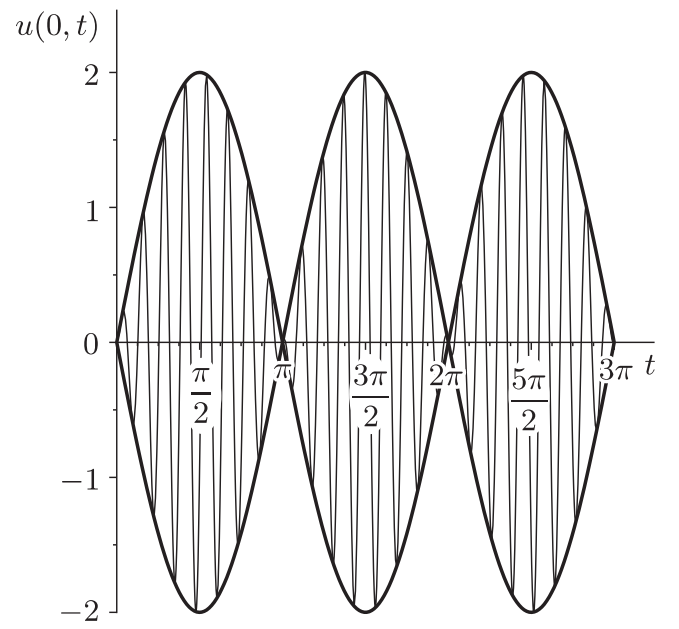

a

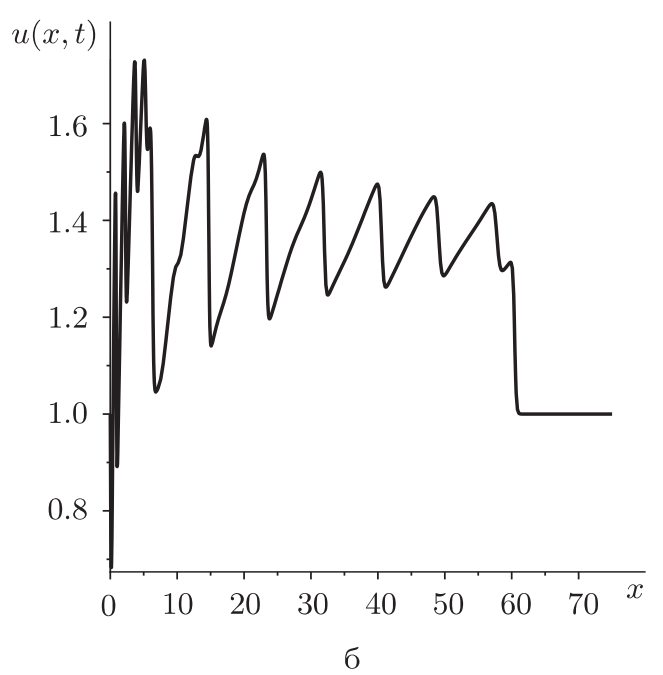

Рис. 3. График возмущения $u(0, t)=1+2 \sin (t) \sin (5 \pi t)$ (а) и решение при $\varepsilon=0.2, t=25$ (б).

Как видно на рис. 2, в той области, где осцилляции угасают, возмущение представляет собой уединенную волну с постоянным профилем и постоянной скоростью, т. е. ведет себя подобно инвариантному по отношению к трансляционной симметрии решению вида

$$
u=-\frac{b}{2 f}-\varepsilon^{2} f \operatorname{th}(c+f x+b t) .
$$

Скорость этой уединенной волны $V=-b / f$. С другой стороны, как можно видеть на графиках, сдвиг вверх для функции гиперболического тангенса равен $\left(A+u_{0}\right) / 2$. Поэтому $V=A+u_{0}$ является оценкой скорости распространения сигнала в среде.

Например, в случаях, представленных на рис. 2 , мы имеем $u_{0}=1, a=2, A \approx \sqrt{3}$ и $V \approx 1+\sqrt{3}$, что хорошо согласуется с численными результатами.

Еще один пример имеет отношение ко взаимодействию между периодическими возмущениями различной частоты. Рассмотрим следующую задачу (здесь $\varepsilon=0.2$ ):

$$
u(0, t)=1+2 \sin (t) \sin (5 \pi t), \quad u(x, 0)=1, \quad u_{x}(75, t)=0 .
$$

Отметим, что $u(0, t)$ - это суперпозиция, $2 \sin (t) \sin (5 \pi t)=\cos (t-5 \pi t)-\cos (t+5 \pi t)$ есть волновой пакет; он представлен на рис. За. Оценка для среднего значения при $t \rightarrow \infty$ в этом случае такова:

$$
\sqrt{\frac{-1}{2 \pi} \int_{0}^{2 \pi}\left(\varepsilon^{2} \frac{d}{d t}(1+2 \sin (t) \sin (5 \pi t))-(1+2 \sin (t) \sin (5 \pi t))^{2}\right) d t} \approx 1.41 .
$$

Заметим, что частота результирующего пилообразного решения, представленного на рис. 3б, является удвоенной по сравнению с огибающей пакета на рис. За (это легко увидеть из рис. $3 б$, поскольку $t=25$ примерно соответствует семи периодам). Этому удвоению частоты есть объяснение: в вязкой среде дольше выживают 


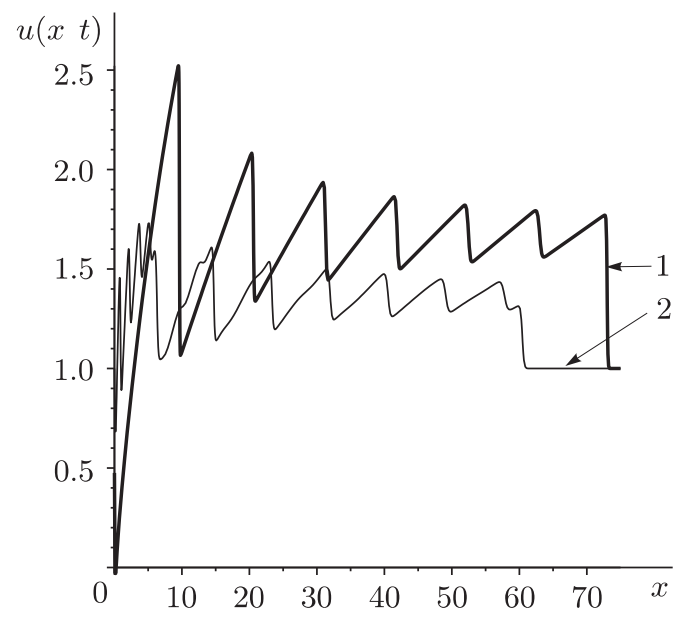

Рис. 4. Решения задач (3) - кривая 1 и (4) - кривая 2.

наибольшие пики, которые определяют профиль решения вдали от источника возмущения, но такие пики возникают в каждом пакете ширины $\pi$, в то время как период огибающей $1+2 \sin t$ есть $2 \pi$.

Однако решение на рис. 3 не стремится к решению, которое получится, если взять в качестве граничного возмущения гармонику с двойной (по отношению к огибающей пакета) частотой.

Решение задачи с начально-граничными условиями

$$
u(x, 0)=1, \quad u(0, t)=1+2 \sin (2 t), \quad u_{x}(75, t)=0
$$

представлено на рис. 4 вместе с решением задачи (3). У последнего из решений более высокий пик, следовательно, возмущение распространяется быстрее: в нашем случае при $t=25$ оно распространилось (примерно) до $x=73$, в то время как первое - только до $x=60$. Кроме того, оценка среднего при $t \rightarrow \infty$ в задаче (4) дает $A \approx \sqrt{3}$, что существенно отличается от $A \approx 1.41$. Подробный анализ подобного рода взаимодействий будет проведен в следующей работе.

\section{4. ВЫВОДЫ И СООБРАЖЕНИЯ О ЧИСЛЕННЫХ РАСЧЕТАХ}

Численное моделирование дает нам бесценную возможность понимания подробностей формирования решений. Графики, приведенные в настоящей статье, были построены при помощи численных расчетов в пакете Maple PDETools; был использован метод Кранка-Николсона.

Следует отметить, что наличие точек разрыва производной внутренне присуще рассматриваемой модели. Однако стандартные численные методы, используемые с параметрами по умолчанию, легко могут терять устойчивость в таких точках. Такая неустойчивость приводит к мультиосцилляциям и общей потере точности. С этой проблемой мы по большей части справлялись, адаптируя (укорачивая) параметры spacestep и/или timestep пакета PDETools. Предметом наших последующих исследований будет качественный анализ взаимодействия периодических возмущений. 


\section{Список литературы}

[1] R. D. Fay, J. Acoust. Soc. Amer., 3 (1931), 222-241.

[2] D. F. Parker, Proc. Roy. Soc. London Ser. A, 369 (1980), 409-424.

[3] О. В. Руденко, УФН, 165:9 (1995), 1011-1035.

[4] A. Samokhin, J. Geom. Phys., 8 (2014), 177-184.

[5] B. Dubrovin, M. Elaeva, Russ. J. Math. Phys., 19:4 (2012), 449-460, arXiv: 1301.7216.

[6] B. Dubrovin, Commun. Math. Phys., 267:1 (2006), 117-139. 